\title{
The Asymptotic Behaviours of a Class of Neutral Delay Fractional-Order Pantograph Differential Equations
}

\author{
Baojun Miao and Xuechen Li \\ School of Mathematics and Statistics, Xuchang University, Xuchang 461000, China \\ Correspondence should be addressed to Baojun Miao; bjmiao2005@126.com
}

Received 1 April 2017; Revised 8 July 2017; Accepted 3 August 2017; Published 22 October 2017

Academic Editor: Antonio Scarfone

Copyright (C) 2017 Baojun Miao and Xuechen Li. This is an open access article distributed under the Creative Commons Attribution License, which permits unrestricted use, distribution, and reproduction in any medium, provided the original work is properly cited.

\begin{abstract}
By using fractional calculus and the summation by parts formula in this paper, the asymptotic behaviours of solutions of nonlinear neutral fractional delay pantograph equations with continuous arguments are investigated. The asymptotic estimates of solutions for the equation are obtained, which may imply asymptotic stability of solutions. In the end, a particular case is provided to illustrate the main result and the speed of the convergence of the obtained solutions.
\end{abstract}

\section{Introduction}

Throughout the past decade, the study of the theory of fractional differential equations has gained a lot of great interests and attracted growing attention. Fractional differential equation has been used successfully in many applied fields such as physics, quantum mechanics, field theory, chemistry, optimal control, and engineering. Hence, the existence and uniqueness of fractional differential equations have been extensively researched by many experts and scholars [17]. It is well known that the pantograph equation arises in quite different fields of pure and applied mathematics and have been investigated extensively [8-10]. Recently, due to its importance in many applied fields and playing an extremely important role in explaining many different phenomena, it is also interesting to study the existence and asymptotic behaviours of the generalized and multipantograph equations. Yüzbasi et al. [8] investigated the numerical solution of generalized pantograph equation with a linear functional argument by virtue of introducing a collocation method based on the Bessel polynomials for the approximate solution of the pantograph equations. Rahimkhani et al. [11] obtained numerical solution of fractional pantograph differential equations by using generalized fractionalorder Bernoulli wavelet. Yu [12] used variational iteration method to solve the multipantograph delay equation, and sufficient conditions were given to assure the convergence of the method. Balachandran and Kiruthika [13] studied the existence of solutions of abstract fractional pantograph equations by using the fractional calculus and fixed point theorems. Peics [14] researched the asymptotic behaviour of solutions of difference equations with continuous argument and obtained asymptotic estimates. Doha et al. [15] using the Jacobi rational-Gauss method reduced solving the generalized pantograph equation to a system of algebraic equations and obtained reasonable numerical results. Motivated and inspired by thoughts and methods of the above recent papers, based on the difference pantograph equations [14], we establish the following neutral delay fractional pantograph equations with continuous arguments of the form

$$
\begin{aligned}
D^{q}[u(t)-A(t) u(t-1)] & =B(t) u(p(t)), \\
u(0) & =u_{0}=0, \\
u(t) & =\varphi(t), \\
&
\end{aligned}
$$

where $0<q<1, A(t)$ and $B(t)$ are all continuous real function and are assumed to satisfy the following two hypotheses throughout this paper:

$$
\left(H_{1}\right) 0<A(t)<1 \text { and } A(0)=0 \text {. }
$$


$\left(H_{2}\right)$ There exists continuous real function $p(t)$ which satisfies the following: when $t>0,0<p(t)<t-\delta(t)$, where $0<\delta^{\prime}(t)<1$ and $\lim _{t \rightarrow \infty} p(t)=\infty$; when $t=0, \delta(0)=0$, which implies $p(0)=0$.

The aim of the paper is to research the asymptotic behaviour of solutions for fractional delay equations with continuous arguments (1), give asymptotic estimates for the speed of convergence of solutions, and apply our results to the particular cases. The paper is organized as follows. In Section 2, we present some notations and definitions of fractional calculus needed in the rest of the paper. Section 3 is devoted to the main results of the paper by using fractional calculus and the summation by parts formula. In Section 4, we obtain the speed of the convergence of the solutions for the initial value problem (1) by using Theorem 3 to deal with special case.

\section{Preliminaries}

For convenience of the reader, we need to introduce some notations and properties of fractional calculus which will be used in the proof of our results. Let $\mathbf{N}$ be the set of natural numbers, and let $\mathbf{R}$ be the set of real numbers and $\mathbf{R}_{+}=$ $(0, \infty)$.

Use the notations

$$
\begin{aligned}
& \prod_{l=t}^{t-1} f(l)=1 \\
& \prod_{t=t-n}^{t} f(l)=f(t-n) f(t-n+1) \cdots f(t) \\
& \sum_{\tau=t}^{t-1} f(\tau)=0 \\
& \sum_{\tau=t-n}^{t} f(\tau)=f(t-n)+f(t-n+1)+\cdots+f(t)
\end{aligned}
$$

for $n \in \mathbf{N}, t \in \mathbf{R}_{+}$and arbitrary real function $f$. The difference operator $\Delta$ is defined by $\Delta f(t)=f(t+1)-f(t)$, where the function $f(t)$ is defined for $t \in \mathbf{R}_{+}$. The difference operator $\Delta_{t}$ is defined by $\Delta_{t} g(t, a)=g(t+1, a)-g(t, a)$, where the function $g(t, a)$ is defined for $a, t \in \mathbf{R}_{+}$.

Let $t_{0}$ be a positive real number and set

$$
\begin{aligned}
t_{-1} & =\min \left\{\inf \left\{p(s): s \geq t_{0}\right\}, t_{0}-1\right\}, \\
t_{n} & =\inf \left\{s: p(s)>t_{n-1}\right\}
\end{aligned}
$$

for $n=1,2, \ldots$. Then $\left\{t_{n}\right\}$ is an increasing sequence such that

$$
\begin{aligned}
& \lim _{n \rightarrow \infty} t_{n}=\infty, \\
& \bigcup_{n=1}^{\infty}\left[t_{n-1}, t_{n}\right)=\left[t_{0}, \infty\right), \\
& p(t) \in \bigcup_{i=0}^{n}\left[t_{i-1}, t_{i}\right), \\
& t_{n} \leq t<t_{n+1}, n=0,1,2, \ldots
\end{aligned}
$$

Fix a point $t$ such that $t_{n} \leq t<t_{n+1}$, define the natural number $k(t)$ such that $t-k(t)-1<t_{n}$ and $t-k(t) \geq t_{n}$, and the set $T(t)$ is defined by

$$
T(t)=\{t-k(t), t-k(t)+1, \ldots, t-1, t\} .
$$

A solution of (1) is a function $u(t)$ which is defined for $t \geq$ $t_{-1}$ and satisfies (1) for $t \geq t_{0}$. For a given real bounded continuous function $\phi$ on $t_{-1} \leq t<t_{0}$, (1) has a unique solution $u(t)$ satisfying the initial condition $u(t)=\phi(t)$.

Definition 1 (see [16]). The Riemann-Liouville fractional integral operator of order $\alpha>0$, of a function $f \in L_{1}\left(\mathbf{R}_{+}\right)$, is defined by

$$
I^{\alpha} f(t)=\frac{1}{\Gamma(\alpha)} \int_{0}^{t}(t-s)^{\alpha-1} f(s) d s,
$$

where $\Gamma(\cdot)$ is the Euler Gamma function.

Definition 2 (see [16]). The Riemann-Liouville fractional derivative of order $\alpha>0, n-1<\alpha<n, n \in N$, is defined by

$$
D^{\alpha}=\frac{1}{\Gamma(n-\alpha)}\left(\frac{d}{d t}\right)^{n} \int_{0}^{t}(t-s)^{n-\alpha-1} f(s) d s,
$$

where the function $f(t)$ has absolutely continuous derivatives up to order $(n-1)$.

\section{Main Results}

In this section, let $M_{0}=\sup _{t_{-1} \leq t<t_{0}} \rho(t)|u(t)|, N_{0}=$ $\inf _{t_{-1} \leq t<t_{0}} \rho(t)|u(t)|$.

Theorem 3. Assume that the conditions $\left(H_{1}\right)$ and $\left(H_{2}\right)$ hold; there exists a real continuous function $\rho:\left[t_{-1}, \infty\right) \rightarrow(0, \infty)$ bounded on the initial interval $\left[t_{-1}, t_{0}\right]$ such that

$$
\begin{aligned}
& \frac{1}{\Gamma(q)} \int_{0}^{t}(t-s)^{q-1} \frac{B(s)}{\rho(p(s))} d s \leq \frac{1-A(t)}{\rho(t-1)} \\
& \text { for } t \in\left[t_{0}, \infty\right)
\end{aligned}
$$

and there exists a real number $R$ such that

$$
\prod_{n=0}^{j}\left(1+R_{n}\right) \leq R, \quad \text { for all positive integers } j
$$

where

$$
R_{n}=\sup _{t_{n} \leq t<t_{n+1}}\left\{\rho(t) \sum_{\tau=t-k(t)}^{t} \frac{\Delta \rho(\tau-1)}{\rho(\tau) \rho(\tau-1)} \prod_{l=\tau+1}^{t} A(l)\right\} .
$$

Assume that $u(t)$ is a solution of the initial value problem (1); then

$$
|u(t)| \leq \frac{M_{0} R}{\rho(t)} \quad \text { for } t \in\left[t_{0}, \infty\right) .
$$


Proof. It is easy to see that (1) is equivalent to the following integral equation:

$$
\begin{aligned}
u(t) & -A(t) u(t-1) \\
= & u_{0}-A(0) u(-1) \\
& +\frac{1}{\Gamma(q)} \int_{0}^{t}(t-s)^{q-1} B(s) u(p(s)) d s
\end{aligned}
$$

that is,

$$
\begin{aligned}
u(t)= & A(t) u(t-1) \\
& +\frac{1}{\Gamma(q)} \int_{0}^{t}(t-s)^{q-1} B(s) u(p(s)) d s .
\end{aligned}
$$

Let $y(t)=u(t) \rho(t)$; then the function $y(t)$ will satisfy the equation

$$
\begin{aligned}
\frac{y(t)}{\rho(t)}= & A(t) \frac{y(t-1)}{\rho(t-1)} \\
& +\frac{1}{\Gamma(q)} \int_{0}^{t}(t-s)^{q-1} B(s) \frac{y(p(s))}{\rho(p(s))} d s .
\end{aligned}
$$

Let $t \in\left[t_{n}, t_{n+1}\right)$ and $\tau \in T(t)$; then (12) is equivalent to

$$
\begin{aligned}
\Delta_{\tau} & \left\{\frac{y(\tau-1)}{\rho(\tau-1)} \prod_{l=t-k(t)}^{\tau-1} \frac{1}{A(l)}\right\} \\
& =\left(\frac{y(\tau)}{\rho(\tau)}-A(\tau) \frac{y(\tau-1)}{\rho(\tau-1)}\right) \prod_{l=t-k(t)}^{\tau} \frac{1}{A(l)} \\
& =\frac{1}{\Gamma(q)} \int_{0}^{\tau}(\tau-s)^{q-1} B(s) \frac{y(p(s))}{\rho(p(s))} d s \prod_{l=t-k(t)}^{\tau} \frac{1}{A(l)} .
\end{aligned}
$$

Summing up both sides of the above equality from $t-k(t)$ to $t$, we get

$$
\begin{aligned}
y(t) & =\frac{\rho(t)}{\rho(t-k(t)-1)} y(t-k(t)-1) \prod_{l=t-k(t)}^{t} A(l) \\
& +\rho(t) \\
& \cdot \sum_{\tau=t-k(t)}^{t}\left\{\frac{1}{\Gamma(q)} \int_{0}^{\tau}(\tau-s)^{q-1} B(s) \frac{y(p(s))}{\rho(p(s))} d s\right\} \\
& \cdot \prod_{l=\tau+1}^{t} A(l) .
\end{aligned}
$$

So from (16), we obtain

$$
\begin{aligned}
& |y(t)| \leq \frac{\rho(t)}{\rho(t-k(t)-1)}|y(t-k(t)-1)| \prod_{l=t-k(t)}^{t} A(l) \\
& \quad+\frac{\rho(t)}{\Gamma(q)} \sum_{\tau=t-k(t)}^{t} \int_{0}^{\tau}(\tau-s)^{q-1} \frac{B(s)}{\rho(p(s))}|y(p(s))| d s \\
& \quad \cdot \prod_{l=\tau+1}^{t} A(l) .
\end{aligned}
$$

Let

$$
\begin{aligned}
\mu_{n} & =\sup _{t_{n-1 \leq t<t_{n}}}|y(t)|, \\
M_{n} & =\max \left\{\mu_{0}, \mu_{1}, \ldots, \mu_{n}\right\} .
\end{aligned}
$$

Since $|y(p(t))| \leq M_{n}$ for $\tau \in T(t)$ and $t_{n} \leq t<t_{n+1}$, by utilizing the summation by parts formula, we can get

$$
\begin{aligned}
& |y(t)| \leq M_{n}\left(\frac{\rho(t)}{\rho(t-k(t)-1)} \prod_{l=t-k(t)}^{t} A(l)+\frac{\rho(t)}{\Gamma(q)}\right. \\
& \left.\cdot \sum_{\tau=t-k(t)}^{t} \int_{t}^{\tau}(\tau-s)^{q-1} \frac{B(s)}{\rho(p(s))} \prod_{l=\tau+1}^{t} A(l)\right) \\
& \leq M_{n}\left(\frac{\rho(t)}{\rho(t-k(t)-1)} \prod_{l=t-k(t)}^{t} A(l)+\rho(t)\right. \\
& \left.\sum_{\tau=t-k(t)}^{t} \frac{1-A(\tau)}{\rho(\tau-1)} \prod_{l=\tau+1}^{t} A(l)\right) \\
& =M_{n}\left(\frac{\rho(t)}{\rho(t-k(t)-1)} \prod_{l=t-k(t)}^{t} A(l)+\rho(t)\right. \\
& \left.\cdot \sum_{\tau=t-k(t)}^{t} \frac{1}{\rho(\tau-1)} \Delta_{\tau}\left(\prod_{l=\tau+1}^{t} A(l)\right)\right) \\
& =M_{n}\left(\frac{\rho(t)}{\rho(t-k(t)-1)} \prod_{l=t-k(t)}^{t} A(l)+\rho(t)\right. \\
& \cdot \frac{1}{\rho(\tau-1)}\left(\left.\prod_{l=\tau}^{t} A(l)\right|_{t-k(t)} ^{t+1}\right. \\
& \left.\left.+\rho(t) \sum_{\tau=t-k(t)}^{t} \frac{\Delta \rho(\tau-1)}{\rho(\tau) \rho(\tau-1)} \prod_{l=\tau+1}^{t} A(l)\right)\right)=M_{n}(1 \\
& \left.+\rho(t) \sum_{\tau=t-k(t)}^{t} \frac{\Delta \rho(\tau-1)}{\rho(\tau) \rho(\tau-1)} \prod_{l=\tau+1}^{t} A(l)\right) \leq M_{n}(1 \\
& +R_{n} \text { ). }
\end{aligned}
$$

From the above inequality, we get

$$
\begin{aligned}
& 1+R_{n} \geq 0, \\
& M_{n+1} \leq M_{n}\left(1+R_{n}\right) \quad \forall n=0,1,2, \ldots .
\end{aligned}
$$

Further, we can obtain

$$
\begin{aligned}
M_{n+1} & \leq M_{n}\left(1+R_{n}\right) \leq M_{n-1}\left(1+R_{n}\right)\left(1+R_{n-1}\right) \\
& \leq \cdots \leq M_{0} \prod_{j=0}^{n}\left(1+R_{j}\right) .
\end{aligned}
$$

Consequently,

$$
|y(t)| \leq M_{0} R
$$

that is, $|u(t)| \leq M_{0} R / \rho(t)$. 
Remark 4. In Theorem 3, suppose that the function $\rho(t)$ is monotone increasing; then the sequence $\left\{R_{n}\right\}$ has only positive members and the assumption

$$
\prod_{n=0}^{\infty}\left(1+R_{n}\right)<\infty
$$

implies the existence of a real number $R$ that satisfies condition (9). If the function $\rho(t)$ is monotone decreasing, then condition (9) is satisfied with $R=1$.

Remark 5. In Theorem 3 , if $B(t)$ is positive continuous real function, then the solution $u(t)$ of the initial value problem (1) is nonnegative for $t \in\left[t_{0}, \infty\right)$.

Theorem 6. If the conditions $\left(H_{1}\right)$ and $\left(H_{2}\right)$ hold, there exists a real continuous function $\rho:\left[t_{-1}, \infty\right) \rightarrow(0, \infty)$ bounded on the initial interval $\left[t_{-1}, t_{0}\right]$ such that

$$
\begin{aligned}
& \frac{1}{\Gamma(q)} \int_{0}^{t}(t-s)^{q-1} \frac{B(s)}{\rho(p(s))} d s \geq \frac{1-A(t)}{\rho(t-1)} \\
& \text { for } t \in\left[t_{0}, \infty\right)
\end{aligned}
$$

and there exists a real number $r$ such that

$$
\prod_{n=0}^{j}\left(1+r_{n}\right) \geq r, \quad \text { for all positive integers } j
$$

where

$$
r_{n}=\inf _{t_{n} \leq t<t_{n+1}}\left\{\rho(t) \sum_{\tau=t-k(t)}^{t} \frac{\Delta \rho(\tau-1)}{\rho(\tau) \rho(\tau-1)} \prod_{l=\tau+1}^{t} A(l)\right\} .
$$

Assume that $u(t)$ is a solution of the positive initial value problem (1); then

$$
u(t) \geq \frac{N_{0} r}{\rho(t)} \quad \text { for } t \in\left[t_{0}, \infty\right)
$$

Proof. It is similar to the proof of Theorem 3. It is seen that (1) is equivalent to the following integral equation:

$$
\begin{aligned}
y(t) & =\frac{\rho(t)}{\rho(t-k(t)-1)} y(t-k(t)-1) \prod_{l=t-k(t)}^{t} A(l) \\
& +\rho(t) \\
& \cdot \sum_{\tau=t-k(t)}^{t}\left\{\frac{1}{\Gamma(q)} \int_{0}^{\tau}(\tau-s)^{q-1} B(s) \frac{y(p(s))}{\rho(p(s))} d s\right\} \\
& \cdot \prod_{l=\tau+1}^{t} A(l) .
\end{aligned}
$$

By utilizing the hypothesis (24), it is easy to see that the function $B(t)$ is positive, by the virtue of Remark 5 , and it implies that the function $y(t)$ is also positive. So we can get the following estimate:

$$
\begin{aligned}
y(t) & \geq \frac{\rho(t)}{\rho(t-k(t)-1)} y(t-k(t)-1) \prod_{l=t-k(t)}^{t} A(l) \\
& +\frac{\rho(t)}{\Gamma(q)} \sum_{\tau=t-k(t)}^{t} \int_{0}^{\tau}(\tau-s)^{q-1} \frac{B(s)}{\rho(p(s))} y(p(s)) d s \\
& \cdot \prod_{l=\tau+1}^{t} A(l) .
\end{aligned}
$$

Define for $n=0,1,2, \ldots$,

$$
\begin{aligned}
v_{n} & =\inf _{t_{n-1 \leq t<t_{n}}}|y(t)|, \\
N_{n} & =\min \left\{v_{0}, v_{1}, \ldots, v_{n}\right\} .
\end{aligned}
$$

Because of $y(p(\tau)) \geq N_{n}$ for $\tau \in T(t)$ and $t_{n} \leq t<t_{n+1}$, by means of the summation by parts formula, we can draw the conclusion

$$
\begin{aligned}
y(t) & \geq N_{n}\left(1+\rho(t) \sum_{\tau=t-k(t)}^{t} \frac{\Delta \rho(\tau-1)}{\rho(\tau) \rho(\tau-1)} \prod_{l=\tau+1}^{t} A(l)\right) \\
& \geq N_{n}\left(1+r_{n}\right) .
\end{aligned}
$$

From the above inequality, it follows that

$$
N_{n+1} \geq N_{n}\left(1+r_{n}\right) \geq N_{0} \prod_{j=0}^{n}\left(1+r_{j}\right) \text {. }
$$

Hence, $y(t) \geq N_{0} r$; that is, $u(t) \geq N_{0} r / \rho(t)$.

Remark 7. In Theorem 6 , if the function $\rho(t)$ is monotone decreasing, then the sequence $\left\{r_{n}\right\}$ defined by (26) is all negative and the assumption $\prod_{n=0}^{\infty}\left(1+r_{n}\right)>0$ implies the existence of a real number $r>0$ which satisfies condition (25). If the function $\rho(t)$ is monotone increasing, then condition (25) is satisfied with $r=1$.

Theorem 8. Assume that conditions $\left(H_{1}\right)$ and $\left(H_{2}\right)$ hold; there exists a real continuous function $\rho:\left[t_{-1}, \infty\right) \rightarrow(0, \infty)$ bounded on the initial interval $\left[t_{-1}, t_{0}\right]$ such that

$$
\begin{aligned}
& \frac{1}{\Gamma(q)} \int_{0}^{t}(t-s)^{q-1} \frac{B(s)}{\rho(p(s))} d s=\frac{1-A(t)}{\rho(t-1)} \\
& \qquad \text { for } t \in\left[t_{0}, \infty\right)
\end{aligned}
$$

and there exist real numbers $r>0$ and $R$ such that conditions (9) and (25) hold for all positive integer $j$. Suppose that $u(t)$ is a solution of the positive initial value problem (1); then

$$
0<\frac{N_{0} r}{\rho(t)} \leq u(t) \leq \frac{M_{0} R}{\rho(t)} \quad \text { for } t \in\left[t_{0}, \infty\right) .
$$

Proof. According to the consequence of Theorems 3 and 6, we can easily obtain the proof. 


\section{Particular Case}

In this section, we use Theorem 3 to deal with special case. By seeking an appropriate function $\rho(t)$, we can also obtain the speed of the convergence of the solutions for the initial value problem (1).

Corollary 9. Let $p(t)$ satisfy $p_{1} t \leq p(t) \leq p_{2}$ for real numbers $0<p_{1} \leq p_{2}<1$. Suppose that conditions $\left(H_{1}\right)$ and $\left(H_{2}\right)$ hold; there exist real numbers $S$ and $\beta$ such that $0<S \leq 1$, $0<\beta<1$,

$$
\begin{aligned}
\frac{1}{\Gamma(q)} \int_{0}^{t}(t-s)^{q-1} \frac{|B(s)|}{\rho(p(s))} d s \leq & \frac{S(1-A(t))}{\rho(p(t))}, \\
\beta \leq & (1-A(t)) \\
& \text { for } t \geq t_{0}>\frac{p_{1}}{1-p_{1}}, \\
\log \frac{1}{S}\left(\log \frac{1}{p_{1}}-\log \frac{1}{p_{2}}\right) & <\log \frac{1}{p_{1}} \log \frac{1}{p_{2}} .
\end{aligned}
$$

Let $u(t)$ be a solution of the initial value problem (1); then

$$
|u(t)| \leq \frac{M_{0} R}{\left(t-p_{1} /\left(1-p_{1}\right)\right)^{k}} \quad \text { for } t \leq t_{0}
$$

where $k=\log S / \log p_{1}, M_{0}=\sup _{t_{-1} \leq t<t_{0}}\left\{\left(t-p_{1} /(1-\right.\right.$ $\left.\left.\left.p_{1}\right)\right)^{k}|u(t)|\right\}$, and

$$
R=\prod_{n=0}^{\infty}\left(1+\frac{k t_{0}^{k}\left(1-p_{1}\right)^{k+1}}{\beta p_{1}^{k}\left(t_{0}\left(1-p_{1}\right)-p_{2}^{n}\right)^{k+1}}\left(\frac{p_{2}^{k+1}}{p_{1}^{k}}\right)^{n}\right)
$$

Proof. By using relations $p\left(t_{n+1}\right)=t_{n}$ and $p_{1} t \leq p(t) \leq p_{2} t$, we can obtain

$$
\frac{t_{0}}{p_{2}^{n}} \leq t_{n} \leq \frac{t_{0}}{p_{1}^{n}} \quad \text { for } n=1,2, \ldots
$$

Let $\rho(t)=\left(t-p_{1} /\left(1-p_{1}\right)\right)^{k}$. Because of $S=p_{1}^{k}$, we have

$$
\begin{gathered}
\frac{1}{\Gamma(q)} \int_{0}^{t}(t-s)^{q-1} \frac{|B(s)|}{\rho(p(s))} d s \leq \frac{S(1-A(t))}{\rho(p t)} \\
\quad \leq \frac{S(1-A(t))}{\rho\left(p_{1} t\right)}=\frac{S(1-A(t))}{p_{1}^{k}\left(t-1 /\left(1-p_{1}\right)\right)^{k}} \\
=\frac{S(1-A(t))}{p_{1}^{k}\left(t-1-p_{1} /\left(1-p_{1}\right)\right)^{k}}=\frac{1-A(t)}{\rho(t-1)} .
\end{gathered}
$$

Consequently, condition (8) is effective. Furthermore, we obtain

$$
\begin{aligned}
R_{n} & =\sup _{t_{n} \leq t<t_{n+1}}\left\{\left(t-\frac{p_{1}}{1-p_{1}}\right)^{k}\right. \\
& \times \sum_{\tau=t-k(t)}^{t} \frac{\left(\tau-p_{1} /\left(1-p_{1}\right)\right)^{k}-\left(\tau-1 /\left(1-p_{1}\right)\right)^{k}}{\left(\tau-p_{1} /\left(1-p_{1}\right)\right)^{k}\left(\tau-1 /\left(1-p_{1}\right)\right)^{k}} \\
& \left.\cdot \prod_{l=\tau+1}^{t} A(l)\right\} \leq \sup _{t_{n} \leq t<t_{n+1}}\left\{t^{k}(1-\beta)^{t}\right. \\
& \left.\cdot \sum_{\tau=t-k(t)}^{t} \frac{k}{\left(\tau-1 /\left(1-p_{1}\right)\right)^{k+1}}\left(\frac{1}{1-\beta}\right)^{r}\right\} \\
& \leq \sup _{t_{n} \leq t<t_{n+1}}\left\{\frac{k t^{k}}{\beta\left(t-k(t)-1 /\left(1-p_{1}\right)\right)^{k+1}}\right\} \\
& \leq \frac{k t_{t_{0}}^{k}}{\beta p_{1}^{k}\left(t_{0}\left(1-p_{1}\right)^{k+1}\right.}\left(\frac{p_{2}^{k+1}}{p_{1}^{k}}\right)^{n} .
\end{aligned}
$$

It follows that Theorem 3 and Remark 4 imply the conclusion.

\section{Conflicts of Interest}

The authors declare that there are no conflicts of interest regarding the publication of this paper.

\section{Acknowledgments}

The present investigation is supported by the Natural Science Foundation of Henan Province (Grant no. 142300410103).

\section{References}

[1] V. Lakshmikantham and A. S. Vatsala, "General uniqueness and monotone iterative technique for fractional differential equations," Applied Mathematics Letters, vol. 21, no. 8, pp. 828834, 2008.

[2] B. Bayour and D. F. Torres, "Existence of solution to a local fractional nonlinear differential equation," Journal of Computational and Applied Mathematics, vol. 312, pp. 127-133, 2017.

[3] X. Yang, Z. Wei, and W. Dong, "Existence of positive solutions for the boundary value problem of nonlinear fractional differential equations," Communications in Nonlinear Science and Numerical Simulation, vol. 17, no. 1, pp. 85-92, 2012.

[4] N. Li and C. Wang, "New existence results of positive solution for a class of nonlinear fractional differential equations," Acta Mathematica Scientia B, vol. 33, no. 3, pp. 847-854, 2013.

[5] H. Zhang, Z. X. Zheng, and W. Jiang, "Existence of solutions for nonlinear fractional-order functional differential equations," Acta Mathematica Scientia, vol. 31, no. 2, pp. 289-297, 2011.

[6] P. Rahimkhani, Y. Ordokhani, and E. Babolian, "A new operational matrix based on Bernoulli wavelets for solving fractional delay differential equations," Numerical Algorithms, vol. 74, no. 1, pp. 223-245, 2017. 
[7] P. Rahimkhani, Y. Ordokhani, and E. Babolian, "An efficient approximate method for solving delay fractional optimal control problems," Nonlinear Dynamics, vol. 86, no. 3, pp. 16491661, 2016.

[8] S. Yüzbasi, N. Sahin, and M. Sezer, "A Bessel collocation method for numerical solution of generalized pantograph equations," Numerical Methods for Partial Differential Equation, vol. 28, pp. 1105-1123, 2012.

[9] S. Yuzbasi and M. Sezer, "An exponential approximation for solutions of generalized pantograph-delay differential equations," Applied Mathematical Modelling, vol. 37, pp. 9160-9173, 2013.

[10] T. Akkaya, S. Yalcinbas, and M. Sezer, "Numeric solutions for the pantograph type delay differential equation using First Boubaker polynomials," Applied Mathematics and Computation, vol. 219, pp. 9484-9492, 2013.

[11] P. Rahimkhani, Y. Ordokhani, and E. Babolian, "Numerical solution of fractional pantograph differential equations by using generalized fractional-order Bernoulli wavelet," Journal of Computational and Applied Mathematics, vol. 309, pp. 493-510, 2017.

[12] Z.-H. Yu, "Variational iteration method for solving the multipantograph delay equation," Physics Letters A, vol. 372 , no. 43, pp. 6475-6479, 2008.

[13] K. Balachandran and S. Kiruthika, "Existence of solutions of nonlinear fractional pantograph equations," Acta Mathematica Scientia B, vol. 33, no. 3, pp. 712-720, 2013.

[14] H. Peics, "On the asymptotic behaviour of difference equations with cintinuous arguments," Dynamics of Continuous, Discrete and Impulsive Systems Series A: Mathematical Analysis, vol. 9, pp. 271-285, 2002.

[15] E. H. Doha, A. H. Bhrawy, D. Baleanu, and R. . Hafez, "A new Jacobi rational-Gauss collocation method for numerical solution of generalized pantograph equations," Applied Numerical Mathematics, vol. 77, pp. 43-54, 2014.

[16] I. Podlubny, Fractional Differential Equations, vol. 198 of Mathematics in Science and Engineering, Academic Press, San Diego, Calif, USA, 1999. 


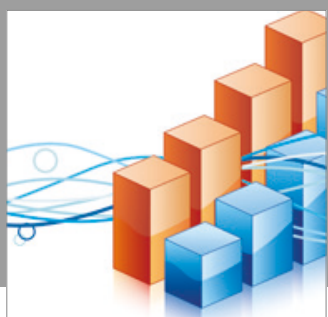

Advances in

Operations Research

vatersals

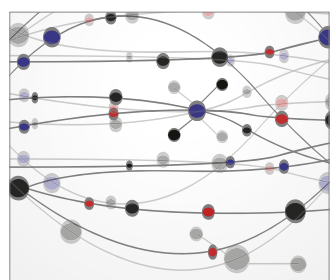

\section{The Scientific} World Journal
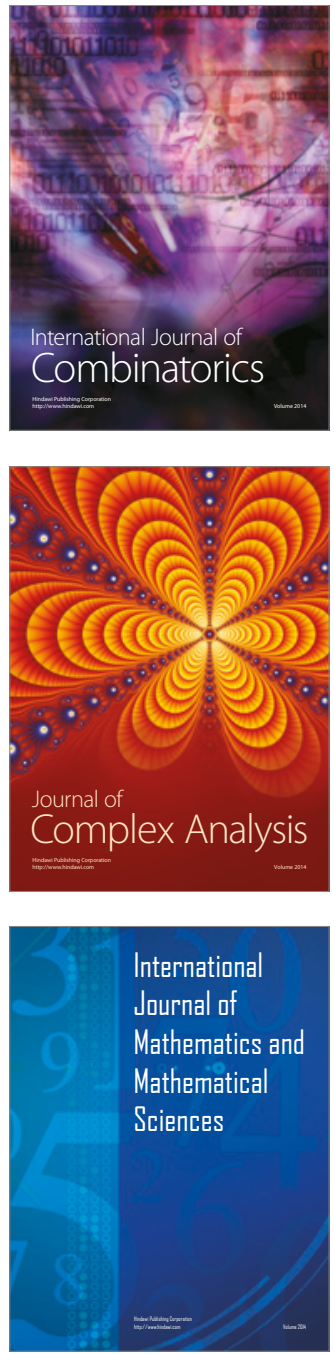
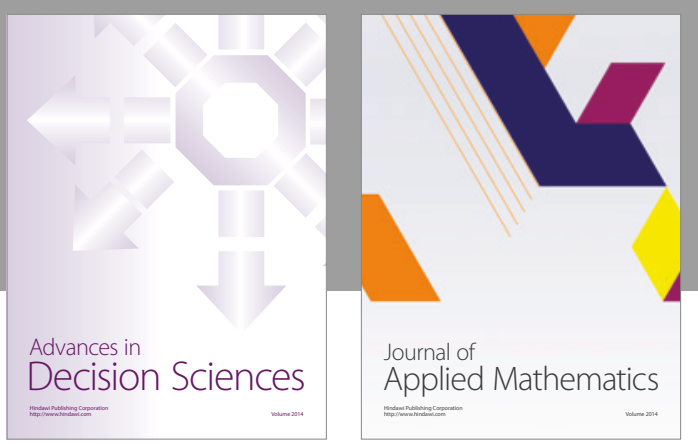

Algebra

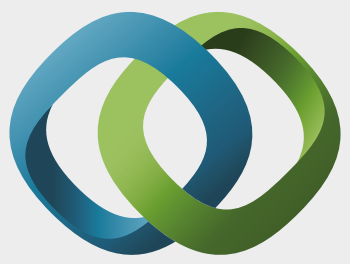

\section{Hindawi}

Submit your manuscripts at

https://www.hindawi.com
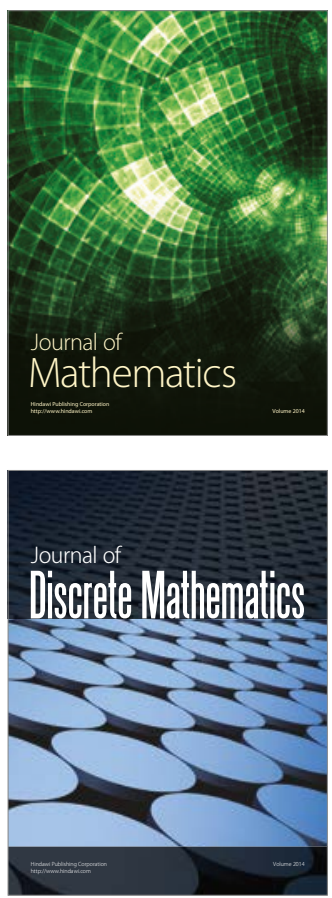

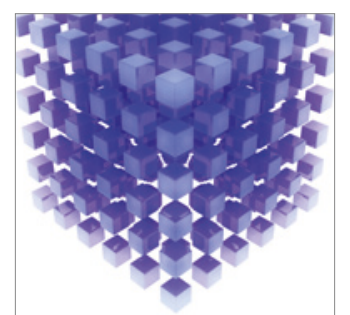

Mathematical Problems in Engineering
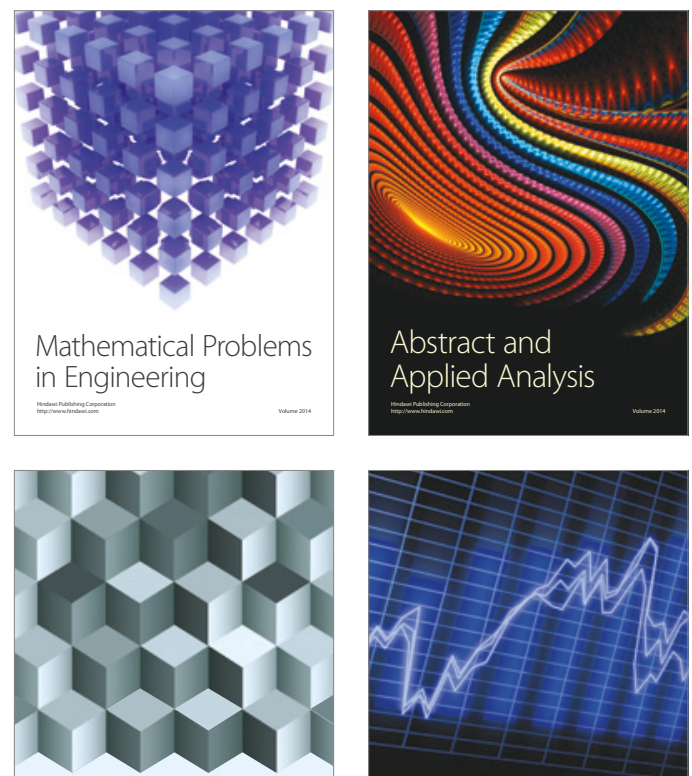

Journal of

Function Spaces

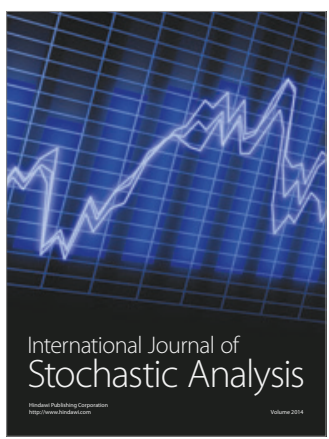

Probability and Statistics
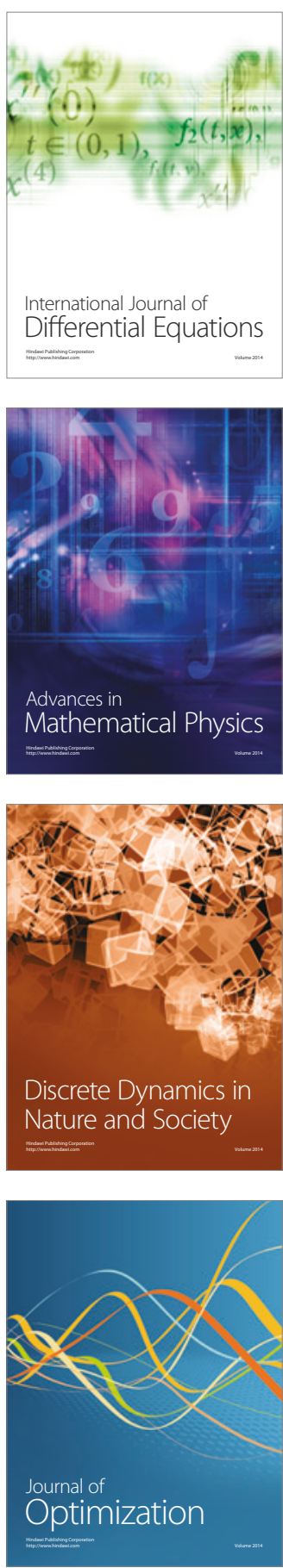